damage. When the researchers blocked key receptors on the neurons, calcium levels subsided somewhat, showing that the damage may be at least partially reversible.

Immunity 33, 424-436 (2010)

\section{OPTICS \\ Through a glass darkly}

A new laser technique can capture an image of an object obscured behind painted glass.

Sylvain Gigan and his team at ESPCI ParisTech in France have devised a method that traces the scattered path that photons take as they pass through an opaque white material. On one side of a glass slide covered in thick white paint, the researchers projected the image of a flower. They illuminated this set-up with a laser and took a photograph from the slide's other side. After calculating the light's zigzagging journey through the painted glass, they were able to reconstruct the flower image.

With improvements, the technique might one day be used in medical imaging to see through opaque biological tissue such as skin.

Nature Commun. doi:10.1038/ ncomms1078 (2010)

\section{DNA replication control}

At the flick of an electronic switch, DNA replication can be turned on or off with the assistance of a nanopore just wide enough for a single DNA molecule to pass through.

Proteins that form molecular pores are important tools for manipulating and studying single molecules of DNA, as well as the polymerase enzymes that bind to DNA and drive its replication. Mark Akeson and his team at the University of California, Santa Cruz, have developed a nanopore system that measures and controls DNA replication as it happens.

The authors designed their system so that polymerase enzymes replicated DNA strands one after the other when the DNA was captured by the nanopore. Reversing the voltage across the pore activated this reaction by shifting the position of the DNA strand within the pore, exposing it to the enzymes. The researchers used their nanopores to measure the rate of replication for hundreds of DNA molecules on the millisecond timescale. Nature Nanotechnol. doi:10.1038/nnano.2010.177 (2010)

\section{COGNITIVE NEUROSCIENCE}

\section{How self-touch relieves pain}

Grabbing hold of an injured area of the body can ameliorate acute pain, but how?

Marjolein Kammers at University College London and her team subjected volunteers to a modified version of the 'thermal grill illusion'. This usually relies on warm and cold bars, but submerging the index and ring fingers in warm water and the middle finger in cool water creates the same sensation - of painful heat in the middle finger.

The volunteers put both hands through this experience. Then, by pressing the three fingers of one hand together with the same fingers on the other hand, volunteers cut their pain level by $64 \%$. Bringing only one or two fingers together, or pressing their fingers against those of the experimenters, did not have this effect. The researchers believe that only full self-contact increases the coherence of the brain's map of the body, which curbs pain.

Curr. Biol. doi:10.1016/j. cub.2010.08.038(2010)

\section{CHEMISTRY \\ Dissolving precious metals}

The scarce metal platinum a valuable catalyst - is hard to recycle if it is mixed with other noble metals such as

COMMUNITY CHOICE

The most read papers in science

\title{
CANCER BIOLOGY
}

\section{Sequencing guides cancer treatment}

\section{HIGHLY READ \\ on genome- \\ biology.com in \\ the last 30 days}

The full genome sequence of a rare tongue tumour has allowed physicians to draw up a personalized treatment plan where no established protocol existed.

A 78-year-old patient had his cancer sequenced after initial treatments failed to slow tumour growth, which had spread to his lungs. When Steven Jones of the British Columbia Cancer Agency in Vancouver, Canada, and his colleagues analysed the sequence and compared it with that of his normal cells, they determined that tumour growth was probably driven by overexpression of a cancer-promoting gene called RET.

A combination of drugs that target RET held back the cancer for seven months, until the tumours became drug-resistant. The team sequenced the cancer again and identified new mutations that had activated the AKT and MAPK pathways, which are often upregulated in cancer. The results provide a snapshot of how tumours evolve to evade treatment.

Genome Biol. 11, R82 (2010)

gold, because the strong acids used to dissolve these metals do so indiscriminately. Wei Lin, Ching-Ping Wong and their colleagues at the Georgia Institute of Technology in Atlanta have come up with a solvent that can efficiently dissolve gold, palladium and silver at room temperature, but leaves platinum untouched.

Varying the composition of the solvent - a mixture of thionyl chloride and organic reagents - allows selective dissolution of metals. The system should help to recover platinum more efficiently from metal waste streams.

Angew. Chem. Int. Edn doi:10.1002/anie.201001244 (2010)

\section{GEOSCIENCE \\ Ocean colour drives storms}

Ocean murkiness depends on the water's level of lightabsorbing molecules such as chlorophyll, and as a result may be a factor in cyclone activity.

A darker ocean absorbs more sunlight, raising sea surface temperatures. Warmer surface waters destabilize the regional atmospheric circulation such that it favours cyclone formation. Using a coupled land-ocean-atmosphere climate model, Anand Gnanadesikan of the National Oceanic and Atmospheric Administration in Princeton, New Jersey, and his colleagues simulated the genesis of subtropical cyclones in the northwestern Pacific. Making non-equatorial regions free from chlorophyll reduced the probability of storm formation over these regions by twothirds, but increased it closer to the equator.

Geophys. Res. Lett. doi:10.1029/ 2010GL044514 (2010)

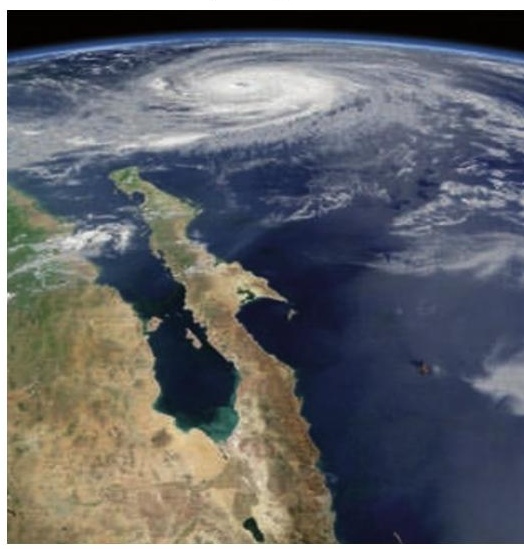

